\title{
Comparison of direct and indirect constraints on anomalous tWb couplings
}

\author{
Anastasiia Kozachuk* \\ D. V. Skobeltsyn Institute of Nuclear Physics, M. V. Lomonosov Moscow State University, \\ 119991, Moscow, Russia \\ E-mail: adkozachuk@gmail.com
}

\begin{abstract}
We analyze constraints on anomalous couplings of the $W t b$ vertex. In particular, we look at scenarios when two of four possible couplings are allowed to vary from their Standard Model values and obtain corresponding indirect constraints from three channels in B-physics: $B_{d, s}-$ $\bar{B}_{d, s}$ oscillations, inclusive decay $B \rightarrow X_{s} \gamma$ and inclusive decay $B \rightarrow X_{s} \ell^{+} \ell^{-}$. We compare our results with constraints of similar scenarios obtained from direct experimental study of the Wtb vertex in the t-channel single top quark production cross section. We conclude that in most twodimensional scenarios indirect constraints are more rigid. So far, no deviations from the Standard Model have been found.
\end{abstract}

European Physical Society conference on High Energy Physics - EPS-HEP 2019,

10-17 July 2019

Ghent, Belgium

${ }^{*}$ Speaker. 


\section{Introduction}

The top quark is the heaviest of all known elementary particles and is expected to have large couplings with physics beyond the Standard Model (BSM physics). One of the possibilities to study BSM physics is to study the anomalous interactions of the $W t b$ vertex. The most general, lowest-dimension, CP-conserving Lagrangian of the $W t b$ vertex has the following form

$$
\mathscr{L}^{W t b}=\frac{g_{w}}{\sqrt{2}} \bar{b} \gamma^{\mu}\left(f_{V}^{L} P_{L}+f_{V}^{R} P_{R}\right) t W_{\mu}^{-}-\frac{g_{w}}{\sqrt{2}} \bar{b} \frac{\sigma^{\mu v} \partial_{v} W_{\mu}^{-}}{M_{W}}\left(f_{T}^{L} P_{L}+f_{T}^{R} P_{R}\right) t+\text { h.c. },
$$

where $P_{L, R}=\left(1 \mp \gamma_{5}\right) / 2, \sigma_{\mu v}=i\left(\gamma_{\mu} \gamma_{v}-\gamma_{v} \gamma_{\mu}\right) / 2, g_{w}$ is the coupling constant of the weak interaction, the form factors $f_{V}^{L, R}$ denote the left- and right-handed vector couplings, and $f_{T}^{L, R}$ denote the left- and right-handed tensor couplings. The Standard Model (SM) has the following set of coupling values: $f_{V}^{L}=V_{t b}, f_{V}^{R}=f_{T}^{L}=f_{T}^{R}=0$. The diagram of the $W t b$ vertex is presented in Fig. 1 .

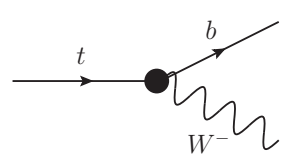

Figure 1: Feynman diagram of the $W t b$ transition. The black circle denotes the vertex which can contain anomalous couplings.

Constraints on anomalous couplings have been studied in several works and can be basically divided into two types. The first type are those obtained from indirect studies of the top interactions in processes involving B-decays. In case of one-dimensional scenarios when only one coupling is allowed to have a non-SM value some of these processes are known to give the most stringent constraints on anomalous $W t b$ couplings $[2,3]$.

The second type are constraints obtained from direct experimental studies of the $W t b$ vertex. Those have been studied at CMS in the t-channel single top quark production cross section [5]. One, two and three dimensional scenarios depending on how many couplings are allowed to have non-SM values have been considered.

In this work we analyzed scenarios with two non-SM couplings, obtained corresponding constraints from three channels in B-physics $\left(B_{d, s}-\bar{B}_{d, s}\right.$ oscillations, inclusive decay $B \rightarrow X_{s} \gamma$ and inclusive decay $B \rightarrow X_{S} \ell^{+} \ell^{-}$) and compared them to similar results from the t-channel single top quark production cross section [5].

\section{Theoretical framework}

We start from the effective Lagrangian containing dimension-six operators. The Lagrangian has the form

$$
\mathscr{L}=\mathscr{L}_{\mathrm{SM}}+\frac{1}{\Lambda^{2}} \sum_{i} C_{i} \mathscr{Q}_{i}+\mathscr{O}\left(\frac{1}{\Lambda^{3}}\right),
$$

where $\mathscr{L}_{S M}$ represents the Standard Model contribution, $\Lambda$ is the scale of New Physics (NP) and $\mathscr{Q}_{i}$ are the dimension-six operators, which are gauge invariant under SM gauge symmetries and 
consist only of SM fields. The scale of NP $\Lambda$ is expected to be large enough so the contribution of new heavy degrees of freedom can be decoupled. In general, when one considers NP contribution as an effective addition to the SM Lagrangian both dimension-five and dimension-six operators are possible [1]. However, in case of $W t b$ interactions dimension-five operators do not contribute since they do not contain quark fields.

In this work we only consider the set of operators which contribute to the anomalous interactions of the $W t b$ vertex:

$$
\begin{aligned}
& \mathscr{Q}_{L L}=\bar{Q}_{L} \tau^{a} \gamma^{\mu} Q_{L}\left(\phi^{\dagger} \tau^{a} i D_{\mu} \phi\right)-\bar{Q}_{L} \gamma^{\mu} Q_{L}\left(\phi^{\dagger} i D_{\mu} \phi\right)+\text { h.c. } \\
& \mathscr{Q}_{R R}=\bar{t}_{R} \gamma^{\mu} b_{R}\left(\tilde{\phi}^{\dagger} i D_{\mu} \phi\right)+\text { h.c. } \\
& \mathscr{Q}_{L R b}=\bar{Q}_{L}^{\prime} \sigma^{\mu v} \tau^{a} b_{R} \phi W_{\mu \nu}^{a}+\text { h.c. } \\
& \mathscr{Q}_{L R t}=\bar{Q}_{L} \sigma^{\mu v} \tau^{a} t_{R} \tilde{\phi} W_{\mu v}^{a}+\text { h.c. }
\end{aligned}
$$

where $\phi$ is the Higgs field, $\tilde{\phi}=i \tau^{2} \phi^{*}, Q_{L}=\left(t_{L}, V_{t b} b_{L}+V_{t s} s_{L}+V_{t d} d_{L}\right)^{T}, Q_{L}^{\prime}=\left(V_{t b}^{*} t_{L}+V_{c b}^{*} c_{L}+\right.$ $\left.V_{u b}^{*} u_{L}, b_{L}\right)^{T}$. After setting the Higgs field in (2.1) to its vacuum expectation value one comes to the Lagrangian of the $W t b$ vertex (1.1).

The anomalous couplings $f_{V}^{L, R}$ and $f_{T}^{L, R}$ from (1.1) are expressed in terms of Wilson coefficients from (2.1) in the following way:

$$
f_{V}^{L}=V_{t b}^{*}+\frac{C_{L L} V_{t b}^{*}}{\sqrt{2} G_{F} \Lambda^{2}}, f_{V}^{R}=\frac{C_{R R}}{2 \sqrt{2} G_{F} \Lambda^{2}}, f_{T}^{L}=\frac{C_{L R b} V_{t b}^{*}}{G_{F} \Lambda^{2}}, f_{T}^{R}=\frac{C_{L R t} V_{t b}^{*}}{G_{F} \Lambda^{2}} .
$$

In this work we only consider the case when the operators in (2.2) are Hermitian and hence the Wilson coefficients (2.1) and the anomalous couplings in (1.1) are real, though the generalization for the complex couplings is, of course, possible.

In processes involving B-decays the constraints on anomalous $\mathrm{Wtb}$ couplings are related to the constraints on the non-SM additions to the Wilson coefficients. The low-energy effective Lagrangian, typically used at the scale $\mu \sim 5 \mathrm{GeV}$ of $b \rightarrow\{s, d\}$ transitions has the following form

$$
\mathscr{L}=\mathscr{L}_{Q C D \times Q E D}+\frac{4 G_{F}}{\sqrt{2}}\left[\sum_{i=1}^{2} C_{i}\left(V_{u b} V_{u s}^{*} \mathscr{O}_{i}^{(u)}+V_{c b} V_{c s}^{*} \mathscr{O}_{i}^{(c)}\right)\right]+\frac{4 G_{F}}{\sqrt{2}} V_{t b} V_{t s}^{*} \sum_{i=3}^{10} C_{i} \mathscr{O}_{i},
$$

where the first term consists of kinetic terms of the light SM particles and their QCD and QED interactions. To obtain the Lagrangian (2.4) from (2.1) one needs to separate the contributions of heavy degrees of freedom of the SM, integrating out the top quark, $W$ and $Z$ bosons, the contribution of which is then becomes encoded in the Wilson coefficients $C_{i}$ of (2.4). (Please, note that the Wilson coefficients in (2.4) are different from those in (2.1).) In this work we do not perform the described calculation, as it was done previously in several works [2,3]. Detailed structure of the operators $\mathscr{O}_{i}$ can be found in [3]. The Wilson coefficients have the form $C_{i}(\mu)=C_{i}^{S M}(\mu)+\delta C_{i}(\mu)$, where the additional term $\delta C_{i}(\mu)$ corresponds to NP contributions.

\section{Constraints on anomalous tWb couplings}

Experimental data on anomalous $W t b$ couplings can be divided into two types: 
- direct constraints, obtained the processes which contain the $W t b$ vertex explicitly, e.g. from the t-channel single top quark production cross section;

- indirect constraints, received indirectly from the channels when the Wtb transition is presented in the loops.

When considering constraints on anomalous couplings different scenarios are possible, depending on how many couplings are allowed to vary from their SM values. One dimensional scenarios, when only one coupling differs from its SM value, are well known [2,3]. In this case, the following three channels give the most stringent constraints on anomalous Wtb couplings:

- data on $B_{d, s}-\bar{B}_{d, s}$ oscillations [4];

- the branching ratio of the $\bar{B} \rightarrow X_{s} \gamma$ decay [2];

- the branching ratio of the $\bar{B} \rightarrow X_{s} \mu^{+} \mu^{-}$decay [3].

Using these three channels, we considered the following scenarios when two or three of the four couplings can have non-SM values: $\left(f_{V}^{L} ; f_{V}^{R}\right) ;\left(f_{V}^{L} ; f_{T}^{L}\right) ;\left(f_{V}^{L} ; f_{T}^{R}\right)$. With these scenarios we obtained the results for indirect constraints from the three channels described above and compared them to direct constraints obtained from the t-channel single top quark production cross section [5]. The results are presented in Fig. 2.

\section{Conclusion}

We performed an analysis of two dimensional constraints when two of the four anomalous couplings $\left(f_{V}^{L}, f_{V}^{R}, f_{T}^{L}, f_{T}^{R}\right)$ can differ from their SM values. In particular, the following scenarios were considered: $\left(f_{V}^{L} ; f_{V}^{R}\right) ;\left(f_{V}^{L} ; f_{T}^{L}\right) ;\left(f_{V}^{L} ; f_{T}^{R}\right)$. For the indirect constraints we considered the following channels in B-physics: data on $B_{d, s}-\bar{B}_{d, s}$ oscillations; the branching ratio of the $\bar{B} \rightarrow X_{s} \gamma$ decay; the branching ratio of the $\bar{B} \rightarrow X_{s} \mu^{+} \mu^{-}$decay. We compared our results with direct constraints of similar scenarios received from t-channel single top quark production cross section [5]. We found that the indirect constraints from B-physics are more rigid than the direct constraints from t-channel single top quark production. So far no deviations from the Standard Model have been observed.

As a prospect, a study of similar three-dimensional constraints with the possibility for three of four anoumalous $W t b$ couplings to vary from their SM values would be interesting.

\section{Acknowledgments}

A.K. was supported by the grant RNF-16-12-10280 of the Russian Science Foundation. A.K. thanks Dmitry Melikhov, Lev Dudko, Dmitry Savin and Petr Mandrik for useful discussions. 


\section{References}

[1] W. Buchmuller and D. Wyler, "Effective Lagrangian Analysis of New Interactions and Flavor Conservation,” Nucl. Phys. B 268, 621 (1986). doi:10.1016/0550-3213(86)90262-2

[2] B. Grzadkowski and M. Misiak, "Anomalous Wtb coupling effects in the weak radiative B-meson decay,” Phys. Rev. D 78, 077501 (2008) Erratum: [Phys. Rev. D 84, 059903 (2011)] doi:10.1103/PhysRevD.84.059903, 10.1103/PhysRevD.78.077501 [arXiv:0802.1413 [hep-ph]].

[3] J. Drobnak, S. Fajfer and J. F. Kamenik, "Probing anomalous tWb interactions with rare B decays," Nucl. Phys. B 855, 82 (2012) doi:10.1016/j.nuclphysb.2011.10.004 [arXiv:1109.2357 [hep-ph]].

[4] J. Drobnak, S. Fajfer and J. F. Kamenik, "Interplay of $t \rightarrow b W$ Decay and $B_{q}$ Meson Mixing in Minimal Flavor Violating Models," Phys. Lett. B 701, 234 (2011) doi:10.1016/j.physletb.2011.05.052 [arXiv:1102.4347 [hep-ph]].

[5] V. Khachatryan et al. [CMS Collaboration], "Search for anomalous Wtb couplings and flavour-changing neutral currents in t-channel single top quark production in pp collisions at $\sqrt{s}=7$ and 8 TeV," JHEP 1702, 028 (2017) doi:10.1007/JHEP02(2017)028 [arXiv:1610.03545 [hep-ex]]. 


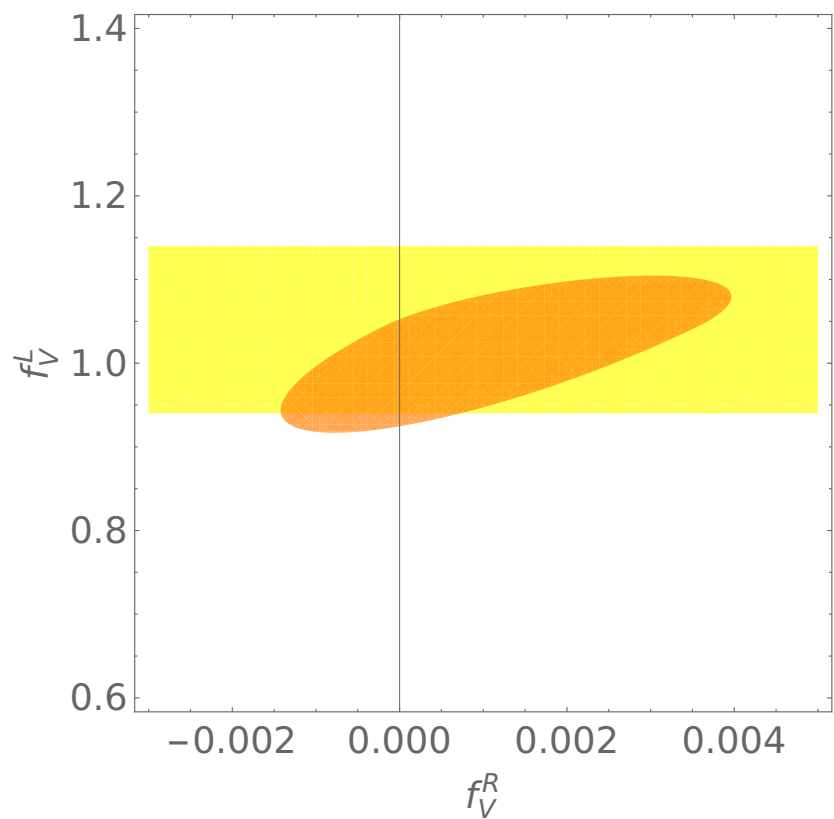

\section{Direct \\ Indirect}

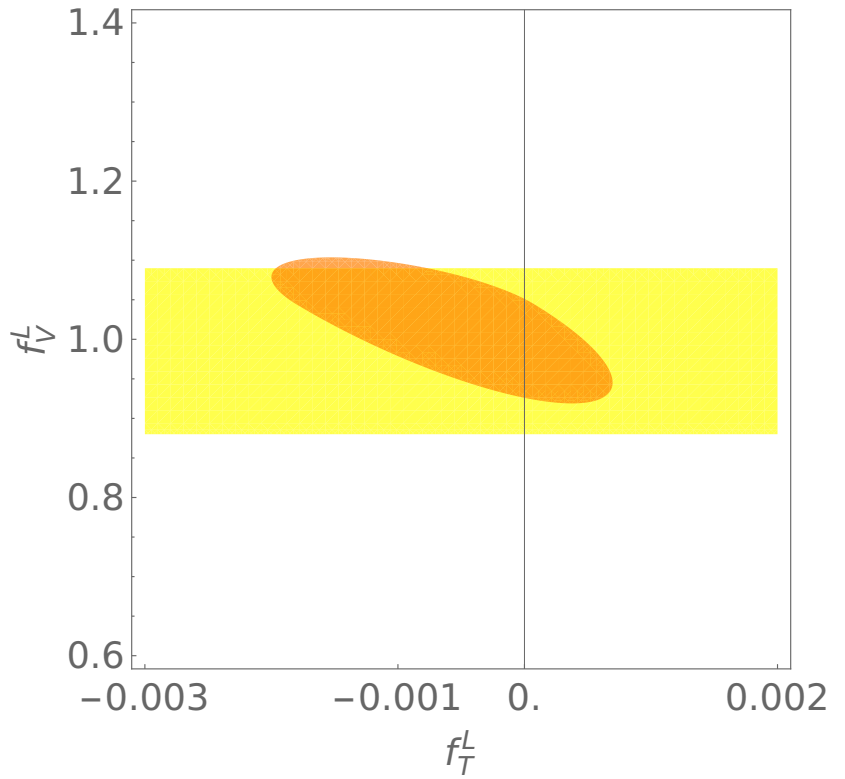

Direct

Indirect

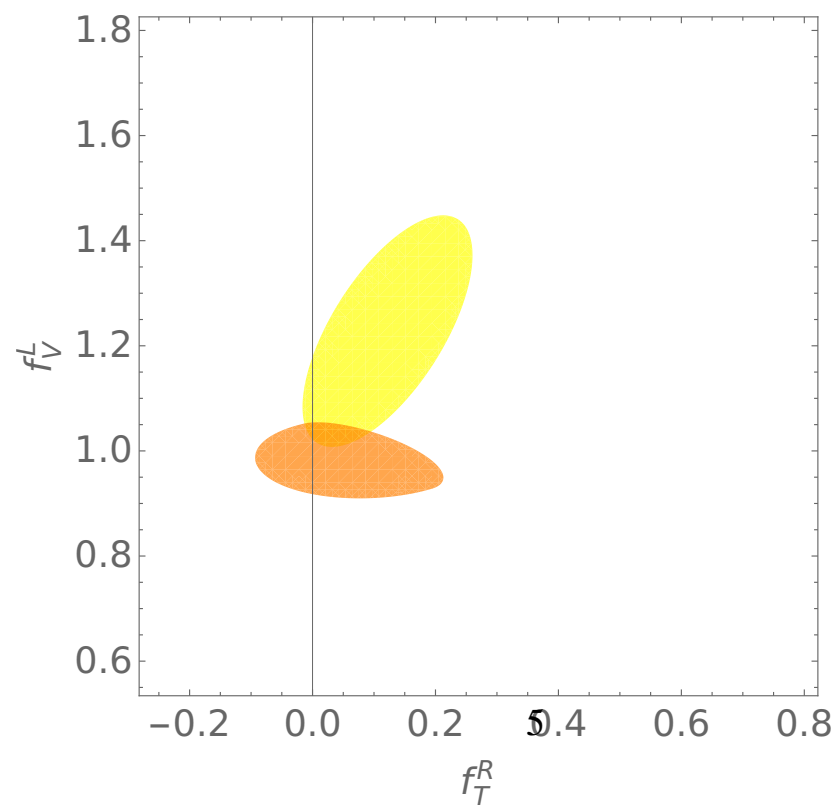

\section{Direct}

Indirect

Figure 2: The images correspond to $95 \%$ C.L. allowed $f_{i}-f_{j}$ regions for indirect constraints from three channels: $B-\bar{B}$ oscillations, $B \rightarrow X_{s} \gamma, B \rightarrow X_{s} \ell^{+} \ell^{-}$; and direct taken from [5]. 